\title{
Isomorphism classes and invariants for a subclass of nine-dimensional filiform Leibniz algebras
}

\begin{abstract}
The paper concerns the classification problem of a subclass of nilpotent Leibniz algebras. This subclass arises from naturally graded non Lie filiform Leibniz algebras. An invarianttheoretic approach to the classification problem of this subclass has been suggested by Rakhimov and Bekbaev. This approach provides to solve the problems with minimum difficulty. Implementing this approach, it is given complete list of isomorphism classes for a subclass of filiform Leibniz algebras and appropriate invariants in dimension nine.
\end{abstract}

Keyword: Invariant; Isomorphism classes; Leibniz algebra 